\title{
XXIV.
}

\section{Beiträge zur Kenntniss der Bildungsfehler der Urogenitalorgane.}

(Aus dem pathol.-anat. Institut der Universität zu Budapest.)

Von Dr. Franz Tangl, zweitem Assistenten.

Es sind einige seltener beobachtete und interessante Missbildungen des Harnapparates, die ich im Folgenden mittheile, von denen zwei mit Missbildungen des weiblichen Geschlechtsapparates combinirt sind. Der eine dieser Fälle (Fall I) bietet auch noch dadurch ein besonderes Interesse, weil es sich um einen Bildungsfehler handelt, welcher bisher noch nicht beobachtet wurde. Wenigstens ist es mir nicht gelungen, einen ähnlichen in der Literatur zu finelen.

Fall I. Congenitale Atrophie und Dystopie der linken Niere. Mündung des linken Ureters in den an beiden Enden blind endigenden persistirenden linken Gartnerschen Kanal. Uterus bilocularis unicollis.

Dieses Präparat fand ich in der Sammlung des Institutes unfertig ausgearbeitet. Aus dem betreffenden Sectionsprotocolle seien folgende Daten angeführt:

E. K., 65 Jahre, Taglöhnerin. Gestorben am 15. November 1887.

Leichendiagnose: Ausgebreitete bochgradige Endarteriitis chronica deformans im ganzen Arteriensysteme. Cbronische Tuberculose der rechten Lungenspitze. Altersemphysem beider Lungen. Chronische interstitielle Entzündung der rechten Niere, mit vielen Retentionscysten. Hypoplasie und Dystopie der linken Niere.

Die Harnorgane sind folgenderweise beschrieben: „Die linke Niere bohnengross, flach, schlaff, weich, $4 \mathrm{~cm}$ über dem Promontorium gelegen. Sie ist mit zwei zwirnfadendünnen Arterien rersehen, deren eine an der Bifurcationsstelle der Aorta, die andere aus der linken Art. jliaca, communis entspringt. Die Vena renal. sinistra mündet in den untersten Theil der Hohlvene. Der linke Ureter tritt mit zwei, je $6 \mathrm{~cm}$ langen Aesten aus dem Hilus der Niere, in deren (d. b. der Aeste) Lumen eine mitteldicke Sonde leicht eingeführt werden kann. Der aus der Vereinigung beider Aeste hervorgegangene Ureter verläuft dann im Bindegewebe hinter der Harnblase. Rechte Niere grauroth, etwas grösser, derb, Oberfläche mit der Kapsel 
stellenweise stärker verwachsen, stark granulirt, mit vielen linsen-bis wallnussgrossen Cysten versehen; Pyramiden stark injicirt. Rechter Ureter normal. In der Harnblase fehlt die linke Uretermündung.“

Ueber die Geschlechtsorgane ist nichts angegeben.

Das Spirituspräparat musste ich weiter ausarbeiten und da stellten sich nach genauer Präparation die Verhältnisse folgenderwaassen dar:

Rechte Niere $11,5 \mathrm{~cm}$ lang, in der Mitte $5,5 \mathrm{~cm}$ breit und $3,6 \mathrm{~cm}$ dick. Im Uebrigen die Zeichen einer interstitiellen Entzündung im Schrumpfungsstadium. Reehter Ureter - vom Ursprung aus dem Nierenbecken gemessen - $26 \mathrm{~cm}$ lang; Nierenbecken mit Kelchen $4 \mathrm{~cm}$. Verlauf und Blasenmündung des Ureters normal.

Linke Niere $2,5 \mathrm{~cm}$ lang, $1,2 \mathrm{~cm}$ breit, $0,5 \mathrm{~cm}$ dick. Plattgedrückte länglich ovale Gestalt; Oberfläche glait. Aus einer hilusartigen Bucht auf einer der breiten Flächen - entspringt der Ureter mit 2 Aesten, die plattgedrückt $4 \mathrm{~mm}$ breit sind und deren Lumen von der Nierensubstanz ausgehend noch eine gauz kurze Strecke obliterirt ist. Nach einem Verlauf von $3 \mathrm{~cm}$ vereinigen sich diese 2 Aeste zu einem ampullenförmig erweiterten Schlauche - plattgedrüekt $11 \mathrm{~mm}$ breit. Diese etwa $3 \mathrm{~cm}$ lange Ampulle setat sich nach unten in einen normal weiten $(5 \mathrm{~mm})$ Ureter fort, dessen Länge $14 \mathrm{~cm}$ beträgt. Der weitere Verlauf des Ureters ist folgender: beilänfig in der Böbe des Ueberganges vom Corpus in den Cervix uteri wendet sich der Ureter zwischen Harnblase und Uterus von binten nach vorne, so dass er etwas über dem Orificium extern. uteri schon auf der vorderen Fläche der Gebärmutter verläuft. Dieser an den Uterus anliegende, an denselben mit Bindegewebe stärker angeheftete Abschnitt des Ureters - circa $6 \mathrm{~cm}$ lang, ist etwas erweitert. Unterhalb dieser erweiterten Strecke verengt sich wieder der Harnleiter, urn nach einem Verlaufe von 2,5 cw, mit einer kleinen beutelförwigen Erweiterung, etwa $3 \mathrm{~cm}$ unterbalb des äusseren Muttermundes blind zu endigen. Dieser letzte $2,5 \mathrm{~cm}$ lange Abschnitt des Harnleiters mit seinem etwas aufgetriebenem Ende ist links in den lateralen Theil der vorderen Scheidenwand eingeschlossen, so dass er ganz in die Muskelsubstanz der Vagina eingebettet ist.

Aus dieser endständigen kleinen Erweiterung des blinden Ureterendes gelangt man mit der Sonde leicht in einen Kanal, der unter spitzem Winkel aufwäts und etwas nach hinten und lateral sich fortsetzt und der - wie es gleich ausfübrlicber beschrieben werden soll, in seinem ganzen Verlaufe in der Wand der Vagina, bezw. des Uterus liegt. Dieser Kanal wurde unter vorsichtiger Führung der Sonde behutsam aufgeschnitten und zeigte im Ganzen folgenden Verlauf: Aus dem erweiterten blinden Ureterende ausgehend zieht er eine $3,5 \mathrm{~cm}$ lange Strecke hinter dem Ureter nach aufwärts und etwas lateral nach rückwärts fast parallel mit der Medianebene des Uterus; dann biegt er unter einem sehr stumpfen Winkel fast gerade nach rückwärts, gegen die hintere Fläche des Uterns gerichtet, so dass dieser 1,2 cm lange Abschnitt des Kanales beinahe einen rechten "Winkel mit der Längsaxe des Uterus bildet; hierauf biegt er wieder nach aufwärts unter einem fast $90^{\circ}$ Winkel um, zu- 
glẹich auch sebr wenig nach vorn gerichtet, um nach einem Verlauf von $1,6 \mathrm{~cm}$ in dieser Richtung, etwa $3 \mathrm{~cm}$ unterbalb des oberen Randes des Fundus uteri, ein wenig zugespitat blind zu endigen. Der ganze mithin $6 \mathrm{~cm}$ lange, durchschnittlich $2 \mathrm{inm}$. weite Kanal ist in seinem Verlaufe also zweimal geknickt und lässt somit 3 Abschnitte: der erste $(3,5 \mathrm{~cm}$ litnge) Abschnitt - von unten an gerechnet - ist anfangs in einer Strecke von etwa $2 \mathrm{~cm}$ Länge, links lateral in der vorderen Wand der Vagina gelegen, in deren Mnskelsubstanz ganz eingebettet, sein oberer Theil $(1,5 \mathrm{~cm})$ liegt aber schon in der Nuskelwand des Uterus, im linken Seitentheile desselben. Ebenso sind auch die übrigen zwei Abschnitte in der Uteruswand gelegen, in ganzen Verlaufe circa $4 \mathrm{~mm}$ unter der Aussenfiäche des linken Uterusrandes. Der zweite Abschnitt ist ganz in der Wand des Cervix, der oberste Abschnitt ragt mit seinem obersten Theile schon etwas in die Wand des Corpus uteri. Unmittelbar unter der ersten Knickung erweitert sich der untere Abschnitt des Kanales - bis zu $5 \mathrm{~mm} \mathrm{-} \mathrm{und} \mathrm{bildet} \mathrm{zugleich} \mathrm{eine}$ kleine Ausbuchtung nach aussen und hinten. An dieser Knickungsstelle selbst ragt aus der hinteren Wand des Kanales ein kleiner quergerichteter leistenförmiger Vorsprung klappenförmig in das Lumen herein. Sonst ist die Innenfläche des Kanales, von einigen minimalen Ausbucbtungen abge. sehen, glatt.

Betrachtet man den Kanal in seinem ganzen Verlaufe, so bildet sein unterster Abschnitt einen sehr fachen, mit der Convexität lateralwärts gekehrten Bogen, mit seinern oberen Ende auch etwas nach rückwärts gewendet, während der oberste Abschnitt ein wenig nach vorn und medianwärts gedreht ist, so dass der ganze Kanal eigentlich etwas spiralig gedreht ist.

Der Uterus $6,5 \mathrm{~cm}$ lang, wovon $3,5 \mathrm{~cm}$ auf den Cervix fallen. Fundus verbreitet, flach $(5 \mathrm{~cm})$, in der Mitte mit einer sehr seichten Vertiefung. Der Cervixkanal ist erweitert. Die Höhle des Uteruskörpers ist durch eine vom Fundus und der hinteren Wand ansgehende Scheidewand in zwei seitliche Hälften getheilt. Die Scheidewand ist im Ganzen dreieckig, mit der Basis im Fundus; nach unten sich allmählich verschmälernd und verdünnend, reicht sie wit ihrer unteren Spitze bis ąn den inneren Muttermund. Das ganze Septum liegt so ziemlich in der Mittellinie, so dass die zwei Kammern des Gebärmutterkörpers mit je einer gleich grossen, für eine mitteldicke Sonde leicht durchgängige Oeffnung in den gemeinsamen Cervixkanal einmünden. In der rechten Kammer ist an der hinteren Wand ein erbsengrosser, breit aufsitzender Schleiwhantpolyp.

Vagina und äussere Genitalien normal.

Es unterliegt wohl keinem $Z$ weifel, dass wir es hier mit einem angebornen Bildungsfehler der linken Niere und des entsprechenden Ureters zu thun haben. - Vor Allem ist der linke Ureter bedeutend kürzer, geht viel tiefer nach unten, als der rechte, der an normaler Stelle mündet. Ausserdem ist auch der 
linke Ureter weiter nach vorne verschoben, so dass sein unterster Abschnitt auf die vordere Wand der Scheide gelangt ist, woselbst er dann blind endigt. Embryonaler Verschluss des uuteren Endes eines Ureters gehört an und für sich nicht zu den sehr seltenen Bildungsfehlern. Was jedoch diesen ganz besonders interessant macht - und ihn von allen bisher beobachteten unterscheidet - ist der Umstand, dass mit dem unteren Ende des linken Ureters noch ein Kanal zusammentrifft, der also an derselben Stelle der Vaginalwand ebenfalls blind endet. (Richtiger ausgedrückt: in dessen untersten blind endenden Abschnitt der Ureter mündet.) Es ist das ein Kanal, den man bei ganz normal entwickelten Geschlechtsorganen - es wird gleich bewiesen, dass er zu diesen gehört - wenigstens makroskopisch nie findet. Die Lage und der Verlauf dieses Kanales rief in mir sofort den Gedanken wach, dass er der persistirte linke Gartner'sche Gang ist, in dessen untersten Abschnitt noch der linke Ureter einmündet. Die nähere Untersuchung, im Vergleiche mit den Ergebnissen der embryologischen Forschung, bestätigten vollkommen meine Voraussetzung.

Es steht fern von mir, hier vielleicht alles das aus der Literatur anführen zu wollen, was über die Gartner'schen bezw. Wolff'schen Gänge geschrieben wurde. Erstens ist die diesbezügliche Literatur schon von Anderen in ihrem ganzen Umfange mitgetheilt worden ${ }^{1}$ ) und zweitens haben wir für uns in erster Reihe jene Untersuchungen Bedeutung, die beim Menschen angestellt wurden. Ich will hier also nur die Resultate aus Dohrn's ${ }^{2}$ ) und Rieder's ${ }^{3}$ ) Arbeit aufzählen. Die übrigen embryologischen Arbeiten will ich hier unerwähnt lassen und verweise diesbezüglich besonders auf Rieder's Arbeit.

Dohrn fand, dass beim menschlichen Embryo die Gartner'schen Gänge nur ausnahmsweise persistiren und da auch nicht in ihrer ganzen Länge; er besitzt niederes cylindrisches Epithel. "Gleich dem Vas deferens zeigt auch der Gartner'sche Gang eine ausgeprägte Neigung zur Schlängelung" besonders im Ligament. latum und in der Uterinsubstanz; im Vaginalgewölbe verläuft er

1) Dohrn, Rieder.

2) Dohrn, Arch. f. Gynäk. Bd.XXI. S. 328.

3) Rieder, Dieses Archiv Bd. 96. 
gestreckt. „Der Gartner'sche Gang erreicht den Uterus in der Gegend des späteren inneren Orificiums, dort bettet er sich ein in den äusseren Rand der concentrischen Muskelschichten des Uterus, liegt oben mehr nach aussen, unten mehr nach vorn und innen." Im Vaginalgewölbe verschwinden seine Spuren gegen die Urethralmündung.

Rieder's Untersuchungen - die neuesten auf diesern Gebiete - erstreckten sich nicht nur auf Embryonen, sondern auch auf Erwachsene.

Nach ihm finden sich: „1) Residuen der Gartner'schen Gänge beim menschlichen Weibe ungefähr in jedem dritten Falle und bleiben bis in's hohe Alter bestehen entweder als ein mit Muscularis umschlossener Epithelschlauch oder als ein Muskelbündel ohne Epithel. Beide Formen sind der Uterus- und Scheidenmuscularis vorn seitlich eingelagert und finden sich häufiger rechts, als links. 2) Der Epithelschlauch ist ausgekleidet mit doppelten, ineinander greifenden, ausnahmsweise mit nur einschichtigen, mittelhohen (durchschnittlich $16 \mu$ ) Cylinderzellen. 3) Das Epithel wird von einer Bindegewebslage und diese von einer Muskelschicht umgeben. Letztere besteht aus glatten Muskelfasern, einer inneren und äusseren longitudinalen und einer mittleren ringförmigen Schicht. 4) In seinem oberen cylindrischen Antheil entspricht der Gang dem Vas deferens; im Bereiche des Cervix, wo die Ausbuchtungen sich finden, der Ampulle und dem Samenbläschen; in seinem Verlaufe durch die Vaginalwand, wo sein Querschnitt spaltförmig erscheint, entspricht er dem Duct. ejaculatorius des Mannes. 5) Im unteren Theile der Vagina ist mir der Gang nie zur Anschaung gekommen, ebenso wenig seine Ausmündung.

Aehnlich lauten auch die Angaben der anderen Forscher. Nur Kocks${ }^{1}$ ) fand am Rande der weiblichen Harnröhre zwei feine Kanäle "mit 2 ganz dicht am hinteren Rande des Orificium urethrae in der Schleimhaut versteckten Oeffnungen". Besonders die Untersuchungen Rieder's haben es aber wahrscheinlich gemacht, dass Kocks' Kanäle, wie es auch schon Dohrn vermuthete, nur Ausstïlpungen der buchtigen Urethralschleimhaut sind.

1) Kocks, Arch. f. Gynäk. Bd. 20. S. 487. 
Die Lage des fraglichen Kanales unseres Falles entspricht vollkommen der Beschreibung Dohrn's und Rieder's. Er verläuft in seiner ganzen Länge in der Scheiden- und Uterusmusculatur: mit seinem unteren Abschnitt seitlich in vorderen Scheidengewölbe, ziemlich oberflächlich, geht dann nach oben unmittelbar in den linken Rand des Cervix uteri über, von allen Seiten von Muskelsubstanz umgeben und reicht mit dem oberen Ende nur etwas ïber, das Niveau des inneren Muttermundes. Der vaginale Abschnitt des Kanales reicht bis zu jener Grenze herab, bis wohin beim Menschen Reste von Gartner'schen Güngen bisher überhaupt gefunden wurden, d. h. im oberen Theile der Vaginalwand in ziemlicher Entfernung von der Mündung der Urethra. - Der ganze Kanal entspricht somit jenem Abschnitte des linken Wolff'schen Ganges, der schon in der Wand des Utero-Vaginalschlauchs verläuft, während jener Theil desselben, der im Ligamentum latum gegen das Ovarium gezogen ist, auch in diesem Falle spurlos verschwunden ist. Der persistirte Abschnitt ist aber in seiner ganzen Länge ohne Unterbrechungen erhalten geblieben und hat am Wachsthum der ihn einschliessenden Organe Theil genommen. Dadurch haben auch seine Dimensionen beträchtlich zugenommen: er hat eine Länge von $6 \mathrm{~cm}$, eine Breite von $2 \mathrm{~mm}$, ja stellenweise sogar von $5 \mathrm{~mm}$. - (Die gewöhnlichen Residuen sind überhaupt nur mikroskopisch nachweisbar.)

Trotz der wesentlichen Zunahme seiner Iimensionen hat der Kanal doch im Allgemeinen die Gestalt beibehalten, die seine Ueberreste gewöhnlich haben. Der Querschnitt seines Lumen ist nicht ganz cylindrisch, der längere Durchmesser ist beiläufig in der Sagittalebene gelegen. (Am Spirituspräparat konnte das nicht mehr sicher festgestellt werden.) Die Neigung Schlängelungen und die verschiedenartigsten Ausbuchtungen zu bilden, ist auch zu bemerken. Wie aus der Beschreibung ersichtlich, ist der Kanal nicht nur 2 mal geknickt - in seinem Cervixtheile -, sondern auch in den einzelnen Abschnitten bogenförmig gekrümmt und ausserdem in der Längsrichtung, etwas spiralig gedreht. Die schon makroskopisch wahrnehmbaren Ausbuchtungen der Wand waren besonders am obersten Theile des unteren Abschnittes sehr ausgesprochen, wo das Lumen noch durch hervorspringende Leisten der Wand unregelmässig wurde. 
Zur mikroskopischen Untersuchung der Kanalwand schnitt ich Stückchen heraus und machte senkrechte Längsschnitte. Die Innenfläche des Kanales ist mit ziemlich hohem, meistens zweischichtigem Cylinderepithel bedeckt, das mit wohl entwickelten Flimmerharen versehen ist. Stellenweise scheint das Epithel nur einschichtig zu sein. An jenen Stellen, wo es zweischichtig, sind die Zellen ganz in der Weise angeordnet, wie es Rieder bei den von ihm gefundenen Gangresiduen beschreibt. In der inneren Schicht sind die kegelförmigen Zellen mit der Basis gegen das Lumen gekehrt; da sitzen auch die Cilien auf. In der zweiten Reihe sind die Zellen niedriger, mit der breiteren Basis nach aussen. Das Epithel ist auf der Oberfläche mit Detritus und braunem oder bräunlichgelbem, grobkörnigem Niederschlag bedeckt, welchen man auch noch in den gleich zu erwähnenden Ausbuchtungen findet. Wahrscheinlich stammen diese Massen aus der Flüssigkeit, die in dem Gange vorhanden war. - Von der inneren Oberfläche gehen zahlreiche, handschuhfingerförmige, sich manchmal verzweigende, drüsenartige Ausstülpungen nach aussen, deren Quer- und Schiefschnitte man in der Schleimhaut des Kanales ziemlich zahlreich vorfindet. Das Epithel dieser Ausbuchtungen ist auf dieselbe Weise beschaffen, wie dasjenige auf der Oberfläche, nur konnte ich hier keine Flimmerhaare mehr sehen. - Unter dem Epithel folgt eine dünne Scbicht ziemlich zellreichen Bindegewebes mit zahlreichen Blutgefässen. Mucosa des Kanales. Diese Mucosa ist von einer Muscularis umgeben, die aus zwei wohl entwickelten longitudinalen Schichten besteht, zwischen welchen eine dünne, circuläre Schicht liegt. Die äussere Längsschicht ist dann durch Biodegewebe von der Uterusmusculatur getrennt. Meine Befunde stimmen vollkommen mit der Beschreibung Rieder's überein. Nur hat Rieder keine Flimmerhaare nachweisen können. Nur Preuschen ${ }^{1}$ ) erwähnt, dass er in den Residuen von Gartner'schen Gängen beim Fuchse Flimmerepithel gefunden hat.

Nach allen diesen Erwägungen ist es, glaube ich, erwiesen, dass der Kanal, in dessen unteres, blindes Ende der linke Ureter mündet, der linke Gartner'sche Gang ist, dessen normale Rückbildung in diesem Falle nicht vor sich gehen konnte. Suchen

1) Preuschen, Dieses Archiv Bd. 70. 
wir nach der Ursache dieser gehemmten Rückbildung, so glaube ich dieselbe in der anomalen Mündung des Ureters in diesen Kanal zu finden.

Bekanntlich entwickelt sich der Ureter aus dem untersten Theile des Wolff'schen Ganges in Form einer kopfwärts wachsenden Ausstülpung seiner hinteren Wand. Es ist dies der Kupffer'sche Nierengang, der sich später vom Wolff'schen Gange abschnürt; um mit einer selbständigen Oeffnung an der hinteren Wand des Sinus urogenitalis zil münden. In unserem Falle ist aus irgend einer jetzt nicht mehr näher bestimmbaren Ursache diese Abschnürung des linken Ureters nicht erfolgt, so dass des Nierenganges ursprüngliche Mündung in den Wolff'schen Gang - der auch als Gartner'sche persistirte - bestehen blieb. Die erwähnte Ursache kann nur eine locale, auf die Nähe der Ureteranlage beschränkte gewesen sein. Vielleicht weist der Umstand, dass in diesem Falle auch im Uterus selbst ein Bildungsfehler vorhanden war - Uterus bilocularis unicollis -, darauf hin, dass jene Prozesse, die die Ursache beider Bildungsanomalien abgaben, möglicher Weise im Funiculus genitalis oder in dessen nächster Umgebung abliefen. - Da sich nun der linke Ureter nicht abschnürte und die Oeffinung des Wolf'schen Ganges in normaler Weise so sich verschloss, mussten beide Kanäle nothwendigerweise nach unten blind enden. Trotzdem am oberen Ende dieses Ureters eine hypoplastische Niere sass, hat deren schwach entwickelte Drüsensubstanz doch ein gewisses Quantum Secret ausgeschieden - wie man das auch thatsächlich bei solchen hypoplastischen Nieren findet ${ }^{1}$ ) - , welches Secret dann durch das untere Ende des Ureters in den communicirenden Gartner'schen Gang gelangt ist und denselben auch angefüllt hat. Aus der Niere konnte so lange Secret in den Ureter gelangen, bis dessen oberes Ende - bezw. die 2 Aeste desselben - nicht obliterirt waren (siehe Beschreibung). Wann diese Obliteration im Laufe des 65jährigen Lebens geschah, kann man freilich nicht mehr angeben. Vielleicht weist die kleine Erweiterung des unteren Endes des Gartner'schen Kanales darauf hin, dass der Secretionsdruck ziemlich lange bestanden hat und genügend war, eine Zeit lang gegen den Druck der Vaginamusculatur zu ) Siehe Fall II. 
wirken. Die in den Gartner'schen Gang stetig zurückgestaute Flüssigkeit gab auch wahrscheinlich das Moment ab, welches die physiologische Obliteration desselben verhindert hat and so die Möglichkeit dazu herstellte, dass der so persistirte Gang am Wachsthum des Utero-Vaginalschlauches Theil nehmen konnte. Es blieb auch nur jener Theil des Wolff'schen Ganges erhalten, der mit den Müller'schen Gängen im Genitalstrang vereinigt verläuft. -

Dieser Fall bildet also das beim Weibe bisher noch nicht beobachtete Analogon zu jener Missbildung des Ureters beim männlichen Geschlecht, wo der Ureter in das Vas deferens, in die Samenblase oder in den Ductus ejaculatorius mündet, wie solche Missbildung auch schon von manchen Beobachtern beschrieben wurde [Hoffmann ${ }^{1}$ ), Rott ${ }^{2}$ ), Boström ${ }^{2}$ ), Weigert $\left.{ }^{3}\right)$ ]. Sehr genau genommen entspräche beim männlichen Geschlecht unserem Falle die Mündung des Ureters in den Ductus ejaculatorius ${ }^{*}$, da aus dem untersten Theile des Wolff'schen Ganges dieser wird ${ }^{5}$ ).

Ich habe schon erwähnt, dass ich in der mir bekannten Literatur keinen ähnlichen Fall gefunden babe; Schrader ${ }^{1}$ ) erwähnt zwar eine Missbildung, wo der Harnleiter in die Vagina mündete, und Förster ${ }^{6}$ ), wo der verschlossene Harnleiter an die linke Seite des Uterus sich ansetzte. Doch in keinem war eine Spur von einem Gartner'schen Kanale.

(Ueber Uterus und Niere gemeinsam mit dem Falle II.)

1) Hoffmann, Arch. f. Heilkunde. 1872.

$\left.{ }^{2}\right)$ Boström, Beiträge zur path. Anat. der Niere. I. Freiburg 1884.

3) Weigert, Dieses Archiv Bd. 104.

4) Eppinger, Klebs Beiträge zur path. Anat. II. Hft. S. 126.

5) Die Erklärung aller dieser Missbildungen bietet seit der wichtigen Kupffer'schen Entdeckung der Ureterentwickelung im Jahre 1866 keine Schwierigkeiten. Thatsächlich berufen sich auch alle Beobachter auf die Kupffer'sche Entdeckung. Nur der Vollständigkeit halber will ich erwähnen, dass, wie ich aus der mündlichen Mittheilung meines hochverehrten Chefs, Herrn Prof. Scheutbauer erfahre, er schon im Jahre 1867 ähnliche Missbildungen des Ureters auf Grund der K u p fer'schen Entdeckung erklärt bat und in seinen Cursen damals in Wien schon auch vorgetragen hat und mithin gewiss unter den allerersten war, die die wichtige Entdeckung auch auf dem Gebiete der pathologischen Anatomie sofort richtig angewendet baben.

6) Förster, Missbildungen u. s. w. 
Fall II. Congenitale Atrophie und Dystopie der linken Niere. Blasenförmige Vorstülpung des linken blinden Ureterendes in die Harnblase. Uterus bilocularis unicollis.

Th. K., 67 Jahre, gestorben am 21. Februar 1889. Klinische Diagnose: Aortenklappeninsufficienz. Morbus Brightii.

Leichendiagnose: Hochgradige Stenose des Aortenostium und Insufficienz der Aortenklappen. Excentrische Herzhypertrophie besonders dẹs linken Ventrikels. Ausgebreitete Atheromatose des Arteriensystemes. Chronische interstitielle und parenchymatöse Nephritis der recbten Niere. Congenitale Atrophie der linken Niere; beutelförmige Vorstülpung des linken blinden Ureterendes in die Harnblase. Uterus bilocularis unicollis.

Die Urogenitalorgane boten bei der Section folgende Verhältnisse:

Rechte Niere $13 \mathrm{~cm}$ lang, $6 \mathrm{~cm}$ breit, $3,5 \mathrm{~cm}$ dick; Kapsel sehwer abziebbar; Oberfäche gelappt und grob granulirt; Corticalsubstanz gelblichgrauroth, derb, geschrumpft, mit vielen schwefelgelben Punkten und Streifen durchsetzt; Pyramiden blutreich. Rechter Ureter $28 \mathrm{~cm}$ lang, mündet an normaler Stelle in die Blase.

Linke Niere $3,5 \mathrm{~cm}$ lang, $1,5 \mathrm{~cm}$ breit, $0,5 \mathrm{~cm}$ dick, liegt um Vieles unterhalb ibrer normalen Stelle, am Psoas; ist länglich-oval, in der frontalen Ebene plattgedrückt, Oberfläche ganz glatt. Von der Aorta gehen zwei dünne, enge, cirea $1,5 \mathrm{~mm}$ breite Arterien zu ihr. Ihre Substanz ist ziemlich consistent, auf der Schnittfläche gelblich-grau, init vielen weissen runden, etwa stecknadelkopfgrossen derben Knoten verseben. Von der hinteren Fläche geht ans einer längsgerichteten Vertiefung der Ureter mit 3 etwa $4 \mathrm{~mm}$ breiten, $3 \mathrm{~cm}$ langen, von einander vollkommen getrennten desten aus - Nierentelche - welche in eine gemeinschaftliche ainpullenförmige Erweiterung - Nierenbecken - (14 $\mathrm{mm}$ breit) äbergeben dessen unteres Ende sich in den in seinem weiteren Verlaufe $6 \mathrm{~mm}$ breiten Ureter fortsetzt. Die ganze Länge des Ureters - die ampullenförmige Erweiterung mitgerechnet - beträgt $16 \mathrm{~cm}$.

Der unterste binter der Harnblase gelegene Abschnitt des Ureters erweitert sich allmählich, durchdringt die hintere Wand der Blase - jedoch nicht so schief wie der rechte Ureter - und geht direct in einen runden, in die Höhle der Harnblase vorspringenden, unter der Schleimhant derselben gelegenen Beutel über. Hebt man die linke Niere in die Höbe, so füllt sich der erwähnte Beutel und wölbt sich als etwa wallnussgrosse kuglige Blase vor. Die Wand des so gefüllten Beutels ist dünn und durchscheinend; die kuglige Oberfläche ist nicht ganz regelmässig, sondern ist in drei kleinere, stärker gewölbte Kugellappen getheilt dadurch, dass die Wand des Beutels einige 1-2 $\mathrm{mm}$ breite Muskelfaserzüge durchziehen, zwischen denen sich die Beutelwandung stärker hervorwölbt. Beide Oberfächen des Beutels sind mit Schleimbaut bedeckt. Bei der Vergleichung der Lage des Bentels mit der Mündungsstelle des rechten Ureters, findet man ersteren, weiter nach unten, zur Urethra näher gelegen. Beim Aufschneiden des linken Ureters 
fliesst aus demselben eine gelblich-braune, etwas trübe, dünne Flüssigkeit. Harnblase mässig contrahirt.

Linke Nebenniere an normaler Stelle und von normaler Grösse.

Aeussere Genitalien und Vagina normal.

Gebärmutter 6,5 cm lang, am Fundus 5,5 $\mathrm{cm}$ breit. Fundus flach, in der Mitte eine sebr seichte Einsenkung. Hintere Fläche des Uteruskörpers stärker convex und trägt in der Mitte in seiner ganzen Länge eine etwas hervorspringende Leiste. Des Canalis cervicalis $(3,5 \mathrm{~cm}$ lang) Längsaxe ist in der Frontalebene ein wenig gekrümmt, mit der Convexität nach links. Mit der Sonde gelangt man nur in die rechte Hälfte des Uterus. Beim Aufschneicen des Uteruskörpers zeigt sich die Höble desselben durch ein Septum in zwei Fäcber getheilt. Das Septum geht vom Fundus und der hinteren Wand aus, ist von dreieckiger Gestalt, mit der Basis im Fundus, nach unten sich allmählieh verschmälernd und verdünnend, verliert sich die untere Spitze desselben in der Nähe des Orificium internum, während sie gleichzeitig von der Medianlinie etwas nach links abweicht. Dadurch ist die rechte Uteruskammer auch etwas weiter und die Mündung des linken in den gemeinsamen einfachen Cervixkanal sehr eng, so dass durch dieselbe nur die allerfeinste Sonde eingeführt werden konnte.

Ovarien geschrumpft, derb, an der Oberfläche stark höckerig, auf der Schnittfäche grau-roth, mit vielen Corporibus albidis versehen.

Es sind in der Literatur im Ganzen nur wenige Fälle von blasenförmiger Vorstülpung des congenital blinden Ureterendes in die Harnblase bekannt. Boströ $\mathrm{m}^{1}$ ) war es, der diese Fälle einer eingehenden Erörterung unterzog und besonders ihre praktische Bedeutung in das richtige Licht stellte. Auch finden sich bei ihm alle bis dahin veröffentlichten ähnlichen Fälle aufgezählt. Unser Fall gehört nicht zu jenen, welche so wie die Boström's, weil sie im Leben gewisse Functionsstörungen hervorriefen, eine praktische Bedeutung hatten. Die vorgestülpte Blase hatte in unserem Falle im Leben absolut keine Functionsstörungen verursacht trotzdem sie auch noch unterhalb der normalen Mündungsstelle des Ureters gelegen war. Bezüglich der Entstehung der vorgestülpten Blase verweise auf die lichtvolle Erklärung Boström's, der ich ganz beipflichten muss. Nur muss man hier annehmen, dass das blinde Ende des Ureters ursprünglich nicht bis unter die Mucosa der Harnblase gereicht haben mag, sobdern von derselben noch durch einige Muskelbündel getrennt war. Wenigstens weisen die plattgewordenen Muskelzüge in der Wand der vorgestülpten Blase darauf hin. Auch werden diese Muskelzüge wahr-

1) Boström, Beiträge zur path. Anat. der Nieren. I. Freiburg 1884. 
scheinlich eine Zeit lang dem Binnendrucke der sich allmählich vorstülpenden Blase entgegengewirkt haben, natürlich waren sie aber zu schwach, um die Vorstülpung ganz zu verhüten. Jedenfalls waren es auch nicht diese Muskelzüge, welche das Nochgrösserwerden der Blase verhindert haben. Wie gross eine solche vorgestülpte Blase wird, hängt in erster Reihe vom Binnendruck, bezw. vom Secretionsdruck der in derselben enthaltenen Flüssigkeit ab. Natürlich müssen ausserdem noch andere günstige Momente da sein, denn sonst könnte eine Vorstülpung überhaupt nicht zu Stande kommen. Das hypoplastische, fast vollständig verödete Nierenparenchym lieferte eine Zeit lang eine gewisse Quantität Secret und schon das Zustandekommen der blasenförmigen Vorstülpung beweist, dass das im Ureter enthaltene Secret unter einem ziemlich hohen Druck gestanden haben mag. (Dieser Umstand beweist auch zugleich, dass solche hypoplastische Nieren mit fast ganz atrophischer Drüsensubstanz noch Secret liefern.) Später wird diese Niere auch ihre schwache Function ganz eingestellt haben - die mikroskopische Untersuchung zeigte eine hochgradige colloide Degeneration in Tubuliresten - und das wird wohl der hauptsächlichste Grund gewesen sein, warum die vorgestülpte Blase nicht noch grösser wurde. Es gehört also dieser Fall zu jenen, welche Boström zu den geheilten rechnet.

Die Uretermissbildung war in diesem Falle, ebenso wie im Falle I, von der Hypoplasie and Dystopie der entsprechenden, also in beiden Fällen der linken Niere begleitet. Es kann kei-

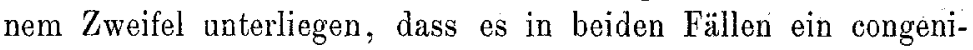
taler Bildungsfehler. ist, dass die Niere auch nie grösser war. Bezüglich der histologischen Structur stimmten beide atrophische Nieren ziemlich überein. In derbem, stellenweise zellreichen und reichlich mit Blutgefässen versehenem Bindegewebe, fanden sich Kanälchen vor, deren zumeist kreisrundes Lumen mit abgelöstem degenerirtem Epithel, meistens aber mit Colloidmassén erfüllt, und deren Innenfläche mit cubischem Cylinderepithel bedeckt ist. $Z$ wischen den Kanälchen sind an manchen Stellen sehr viel Kalkconcretionen abgelágert - besonders im Fall I. Die Kanälchen sind die Ueberreste der mangelhaft entwickelten und degenerirten Harnkanälchen. Im Bindegewebe zerstrent waren noch grosse runde, bezw. kuglige Gebilde, die selbst auch aus Binde- 
gewebe bestanden, fast homogen aussahen; nur an einigen sah man undeutlich eine gewundene Linienzeichnung. Es sind das die obliterirten Glomeruli. An den grösseren mikroskopischen Arterien war eine ziernlich ausgesprochene productive Endarteriitis. In keinem dieser beiden Fälle zeigte die andere Niere eine bedeutende compensatorische Hypertrophie. Die Ursache davon ist die vorgeschrittene interstitielle Nephritis, welche schon in das Schrumpfungsstadium getreten ist und so das Volumen der jedenfalls grösseren Nieren schon verkleinert hat.

Auch im Uterus war bei Fall I und II derselbe Bildungsfehler: Uterus bilocularis unicollis. Beide Uteri boten an und für sich dasselbe Verhalten, wie es schon $\mathrm{Kussmaul}^{1}$ ) und Rokitansky ${ }^{2}$ ) beschrieben haben - Neues kann ich nicht dazufügen. Ich will nur bezüglich der Combination, in welcher dieser Bildungsfehler des Uterus in unseren beiden Fällen auftrat, einige Bemerkungen machen. Schon Rokitansky hat darauf aufmerksam gemacht, dass man zuweilen bei Uterus unicornis, bei $\mathrm{U}$. bicornis und $\mathrm{U}$. bilocularis, Mangel einer Niere findet. In unseren Fällen war wohl nicht Mangel einer Niere, aber eine Missbildung der linken Niere vorhanden. Bedenkt man den engen Zusammenhang zwischen der Entwickelung der Harn- und Geschlechtsorgane, so sind solche Combinationen von Bildungsfehlern leicht begreiflich. Was speciell den Umstand betrifft, dass die erwähnten Hemmungsbildungen des Uterus von Mangel, bezw. Missbildungen einer Niere öfter begleitet sind, glaube ich, dass das vermittelnde Moment $z$ wischen beiden Missbildungen immer ein gleichzeitig bestehender Bildungsfehler des entsprechenden Ureters ist. Ueberbaupt sind ja fast alle Missbildungen und congenitalen Affectionen der Niere von einem mehr oder minder ausgeprägten Bildungsfehler des Ureters begleitet, was aus dem innigen Verhältniss, welches zwischen der Entwickelung der Niere und des Ureters besteht, selbstverständlich ist. Ebenso ist die Coincidenz der erwähnten Hemmungsbildungen des Uterus und der Uretermissbildungen leicht verständlich.

Die vollkommene Abschnürung des Creters vom Urnierengange und die Bildung seiner selbständitgen Oefinung am Sinus

1) Kussmaul, Von dem Mangel, der Verkümmerung u.s.w. der Gebärmutter u.s.w. Würzburg 1859 .

3) Rokitansky, Lehrbuch d. path. Anat. 3. Aufl. II. Bd. 
urogenitalis geschieht beiläufig in derselben Zeit, als zwischen dem im Funiculus genitalis gelegenen Abschnitt der Müller'schen Gänge die Scheidewand zu schwinden und der Fundus sich $\mathrm{zu}$ bilden anfängt. Wenn nun zu jener Zeit ans irgend einer Ursache gewisse Vorgänge in der Umgebung jener Gebilde abliefen, können sie sehr leicht sowohl die normale Involution des Uterusseptum, als die normale Entwickelung des untersten Ureterabschnittes stören. (Speciell auf unsere 2 Fälle mag diese Auffassung wohl anwendbar sein.) Dies kann um so leichter geschehen, da sich der abschnürende und an die vordere Seite des Urnierenganges gelangende Ureter mit seinem unteren Stücke in unmittelbarer Nähe der Müller'schen Gänge sich befindet.

Fall III. Beiderseits gespaltene Ureteren. Gemeinsame Blasenmündung der rechten Ureteren. Tiefere Mündung des linken unteren Ureters in die Harnblase. Dilatation und Mündung des linken oberen Ureters in die Pars prostatica urethrae. Hydronephrosederoberen Hälfte der linken Niere.

Dieses Präparat fand sich ebenfalls in der Sammlung des Instituts und musste noch präparirt werden. Das Sectionsprotocoll lieferte folgende Daten:

Johann Hulek, 28 Jabre. Klin. Diagn.: Delirium tremens. Section am 24. April 1884.

Leichendiagnose: Pachymeningitis haemorrbagica acuta. Hyperaemia cerebri. Sclerosis cranii. Nepbritis parenchymatosa et interstitialis chronica. Ureter duplex bilateralis. Hydronepbrosis majoris gradus partis superioris renis sinistri cum dilatatione ureteris superioris sinistri per prostatam in urethram inosculantis.

Die Beschreibung der Harnorgane ist sehr dürftig und mangelhaft; es istnur die Hydronephrase oberflächlich beschrieben, über die Ureteren ist ausser der Erweiterung des rechten oberen nichts gesagt. Es wäre überflüssig das zu citiren.

Am Spirituspräparat fanden sich folgende Verhältnisse:

Rechte Niere $12,5 \mathrm{~cm}$ lang, $6 \mathrm{~cm}$ breit, $3,5 \mathrm{~cm}$ dick. Oberfläche etwas grob granulirt. Im Hilus sind 2 Nierenbecken, von welchen das obere grösser und weiter und auch reichlicher mit Fett versehen ist. Aus den von einander vollkommen getrennten Becken geht je ein Ureter aus, von denen der obere $21 \mathrm{~cm}$, der untere $17 \mathrm{~cm}$ lang ist, die sich inten zu einem gemeinsamen $8,5 \mathrm{~cm}$ langen Schlauche vereinen der nicht breiter ist als die getremnten Ureteren $(5-7 \mathrm{~mm}$ plattgedrückt) und der an der normalen Stelle der rechten Ureteröffuung in die Harnblase mündet.

Linke Niere $17 \mathrm{~cm}$ lang, $7 \mathrm{~cm}$ breit, $3,5 \mathrm{~cm}$ dick. Hilus stark gegen die hintere Oberfläche verschoben. Der obere Theil der Niere ist zu einer 
faustgrossen (Durchmesser $9 \mathrm{~cm}$ ) Cyste umgewandelt, deren einige Millimeter dicke Wand peripherwärts - (d. i. vis-à-vis der Abgangsstelle des Ureter) dicker und theilweise noch aus atrophischer Nierensubstanz bestehend ist, die sich allmäblich in die Wand des Nierenbeckens verliert, welches den unteren Theil der Cyste bildet. Die Innenfläche des Sackes zeigt noch kleine Reste der atrophirten Pyramiden in Form breiter, etwas hervorspringender Leisten. Der untere, dem erweiterten Becken entsprechende Theil des Sackes ist an der Innenfäche glatt. Die untere Bälfte der Niere zeigt keine Spur ron Eydronephrose, hat ein eigenes Becken, etwa so gross wie ein normales, welches von dem erweiterten des oberen hydronephrotischen Nierentheiles ganz getrennt ist. Dieses untere Nierenbecken liegt fast ganz frei im Hilus, von Nierensubstanz gar nicht verdeckt. Beide Nierenbecken besitzen je einen Ureter. Der aus dem oberen Becken entspringende Ureter ist beiläufig zur Fingerdicke erweitert, erweitert sich in seinem mittleren Theile noch mehr, bis zu $2,5 \mathrm{~cm}$ (plattgedrückt), wird dann weiter nacb unten wieder etwas enger. Statt an der normalen Stelle an den Blasengrund zu treten, verläuft er nach unten zum oberen Rande der linken Prostatabälfte; bis zu diesem Punkte hat er eine Länge von $31 \mathrm{~cm}$, und war in diesem Verlaufe - am Spirituspräparate konnte man es nicht mehr sicher feststellen - wahrscheinlich auch geschlängelt. Vom oberen Rande der Prostata geht er dann zwischen Harnblase und Drüse weiter nach unten und etwas nach vorn, so dass der hintere grössere Theil seiner Perjpherie ron Prostatasubstanz umgeben ist, während seine vordere Fläche mit der Wand der Harnblase bezw. der Urethra in Berührung steht. Dieser in die Prostata eingebettete, bis zu seiner Uretbralmündung 2,5 $\mathrm{cm}$ lange Uretertheil, biegt mit seinem unteren Theile etwas nach rorn, spitzt sich conisch zu und mündet mit einer bleinen ovalen - Durchmesser etwa $1 \mathrm{~mm}$ Oeffnung in die Urethra, $3 \mathrm{~mm}$ oberhalb des linken Randes des Colliculus seminalis, etwas lateralwärts verschoben, so dass die Oeffnung etwas ausserbalb der linken Seite des Trigon. Lieutaudii liegt. Um die kleine Oeffnung bildet die Schleimbaut eine kleine halbmondförmige, mit der Concavität abwärts gekehrte. Falte. Dieser prostatische Theil des Ureters liegt lateral und etwas nach vorn von der linken Samenblase und dem entsprechenden Duct. ejaculatorius und communicirt weder mit dieser noch mit dew Vas deferens, wie das eine sorgfältige Präparirung sichergestellt hat.

Der aus den linken unteren Nierenbecken entspringende Ureter ist bedeutend kürzer und enger als der eben bescbriebene: $26 \mathrm{~cm}$ lang und $4 \mathrm{~mm}$ breit (plattgedrückt). Nach unten verläuft er zum Blasengrunde um in die Harnblase zn wünden und zwar unterhalb der normalen Mündungsstelle; in Folge dessen ist das Trigon. Lieutaudii ein ungleichseitiges Dreieck, dessen rechte Seite $33 \mathrm{~mm}$, die linke nur $28 \mathrm{~mm}$ lang ist.

Beide Vasa deferentia und Samenblasen sind ganz normal. Die Ductus ejaculatọrii münden getrennt in die Vesicula prostatica.

In der Urethra ausser der oben beschriebenen Uretermündung nichts Abnormes. 
Verdoppelung beider Ureteren gehört nicht zu den seltenen Bildungsfehlern. Auch ist das Verhalten der rechten Ureteren in unserem Falle das Gewöhnlichere. Besonderes Interesse bieten nur die linken Ureteren. Sie münden getrent und beide an anormaler Stelle: der eine in die Harnblase, der andere in die Urethra. Der zur oberen Nierenhälfte gehörende Ureter mündet tiefer und medianer (in die Urethra), als der zur unteren Nierenhälfte gehörende. Das gegenseitige Verhältniss der 2 linken Ureteröffnungen entspricht also der von Weigert ${ }^{1}$ ) aufgestellten Regel, dass bei gespaltenen Ureteren mit getrennten Oeffnungen der obere jmmer tiefer und medianer, der untere höher und lateraler mündet. Wahrscheinlich fand in unserem Falle auch eine Kreuzung der linken Ureteren statt, nur war das am Spirituspräparat nicht mehr ersichtlich. Die Mündung des linken unteren Ureters war insofern anormal, als sie ziemlich tiefer lag als die rechte Uretermündung. Der obere linke Ureter mündete ganz selbständig oberhalb und etwas nach links vom Colliculus seminalis und trat mit den benachbarten Organen absolut nicht in Verbindung, war aber mit keiner Anomalie der Geschlechtsorgane combinirt. (Dass die Duct. ejaculatorii in die Vesicula prostatica münden, gehört doch nur zu den selteneren Varietäten.) Wir müssen aber zur Erklärung annehmen, dass bei den doppelt angelegten Nierengängen, was wahrscheinlicher ist - oder auch vielleicht bei den ursprünglich einfachen und sich nur später der Länge nach theilenden Nierengang - einerseits das Zwischenstück zwischen der Mündung des linken Wolff'schen Ganges und des späteren oberen Ureters ungenügend gewachsen ist, wodurch die Oeffung der letzteren nicht genügend aufwärts hat rücken können, wodurch auch wahrscheinlich das Hinaufrücken des (später) unteren Ureters beeinträchtigt wurde. Der Verlauf des unteren Stückes des oberen Ureters in der Prostata macht auch das Zustandekommen der Hydronephrose der entsprechenden Nierenhälfte verständlich, wenn man mit Weigert annimmt, dass die Prostata als musculöses Organ Sphincterendienste verrichtete und so zeitweise Verengerung oder auch vollständige Compression veranlasste. Dies genügte, um im Ureter Stauung des Secretes hervorzurufen, was zuerst 1). Weigert, Dieses Archiv Bd. 70. 
die Erweiterung und die Dehnung, also Verlängerung desselben zur Folge hatte und später die hochgradige Erweiterung des Nierenbeckens und der -kelche und die hydronephrotische Atrophie des Nierenparenchyms nach sich zog. (Im Leben bestand wegen der Mündung des einen Ureters in die Urethra kein Harnträufeln oder ein ähnliches Symptom. In der Krankengeschichte sind von der Function der Harnorgane keine Abnormitäten verzeichnet.)

Diesem Falle ähnlich, doch nicht übereinstimmend, ist ein Fall Boström's ${ }^{1}$ ) und der von Heller ${ }^{2}$ ) angeführte Walter's.

Schon der Umstand, dass derselbe oder wenigstens ähnliche Bildungsfehler des Ureters in einem Falle von der Hypophasie, im anderen von der Hydronephrose (congenitale) der entsprechenden Niere oder Nierenhälfte begleitet sind, beweist, dass der Bildungsfehler des Ureters allein nicht genügt, dieses verschiedene Verhalten der Nierensubstanz zu erklären. Beim Durchsehen der in der Literatur angeführten Beobachtungen wollte es mir Anfangs scheinen, als ob in jenen Fällen, wo der Ureter nicht blind endigte, sondern nur verengt oder einer zeitweiligen Compression ausgesetzt ist, oder allgemeiner ausgedrückt, wenn es im Ureter nur zu temporärer Harnstaunng kommt - ich spreche nur vom embryonalen Leben - Hydronephrose auftrete; während bei gänzlichem Mangel einer Uretermündung, ohne jede Communication mit einem anderen eine Oeffnung besitzenden Organ; Hypoplasie (congenitale Atrophie) der Niere sich entwickelte. Diese Vermuthung rechtfertigte sich nicht, jedenfalls lässt es sich nicht als Regel aufstellen, denn es giebt Fälle, die dieser Regel nicht entsprechen.

Nach den neuesten embryologisehen Untersuchungen entwickelt sich die Niere aus zwei verschiedenen Anlagen, die erst secundär" zu einander in Beziehung treten ${ }^{3}$ ): die Tubuli recti entwickeln sich aus dem Nierengange, dem Ureter, die gewundenen Kanäle, sowie die Henle'schen Schleifen aus einer besonderen Anlage. Diese letztere Nierenanlage erführt eine bedeutende Lageveränderung nachdem der Harnleiter in sie eingedrungen

1) Boström, a. a. 0 .

3) Heller, D. Arch. f. klin. Med. Bd. V.

3) 0. Hertwig, Lehrb. d. Entwickelungsgesch. Jena 1888. S. 272. 
ist und wird durch den wachsenden Ureter weiter nach vorn geschoben und erst in der neuen Lage stellen sich in ihr die inneren Differenzirungen ein.

Es ist also für die weitere Entwickelung der Niere von höchster Wichtigkeit, ob und wie die Verbindung dieser beiden Anlagen gelingt. Es ist nicht unwahrscheinlich, dass eine Entwickelungsstörung am unteren Ende des Ureters als prädisponirendes Moment auch füi' die Entwickelung des oberen Ureterendes, also auch eines Theiles der Niere, sich sehr früh geltend macht. Es wäre nun nicht unmöglich, dass in jenen Fällen, wo die Verbindung der beiden Anlagen nicht oder nur sehr mangelhaft gelingt, also auch die innere Differenzirung in der Nierenanlage ev. nur eine sehr mangelhafte werden konnte, sich die Hypoplasie der Niere einstellt, die auch fast immer mit der Verkürzung des Ureters verbunden ist. Hat die Verbindung der beiden Anlagen stattgefunden, und ist auch die innere Differenzirung vor sich gegangen, und konnte in Folge dessen die Niere hier auch genügend Secret liefern, dann stellt sich, in Folge des fehlerhaften Ureters, Hydronephrose ein. Ich will jedoch damit nicht mehr als eine Vermuthung ausgesprochen haben. Auch will ich damit nicht in Abrede stellen, dass dem Blutgefässapparat eine wichtige Rolle zufällt und es gewiss von Bedeutung ist, dass die erwähnten Bildungsfehler der Niere gewöhnlich von Anomalien der Blutgefässe begleitet sind. Doch kann man aus diesen allein nicht die Missbildung erklären, giebt es doch viele normale Nieren mit grossen Gefässanomalien ${ }^{1}$ ). Auch darf man nicht vergessen, dass die mangelhafte Entwickelung der Gefässe erst die Folge der mangelhaften Entwickelung der Nieren sein kann. Jedenfalls steht der Bildungsfehler des Ureters in näherem causalen Zusammenhange mit den erwähnten Missbildungen der Niere, als die Anomalie der Blutgefässe. Vielleicht wird die genauere mikroskopische Untersuchung einer grösseren Anzahl von hypoplastischen und congenital hydronephrotischen Nieren vou verschiedenem Alter Aufschluss über diese Frage geben und für die oben ausgesprochene Vermuthung — oder auch für eine andere Hypothese - Anhaltspunkte liefern.

1) Boström, a. a. O. 